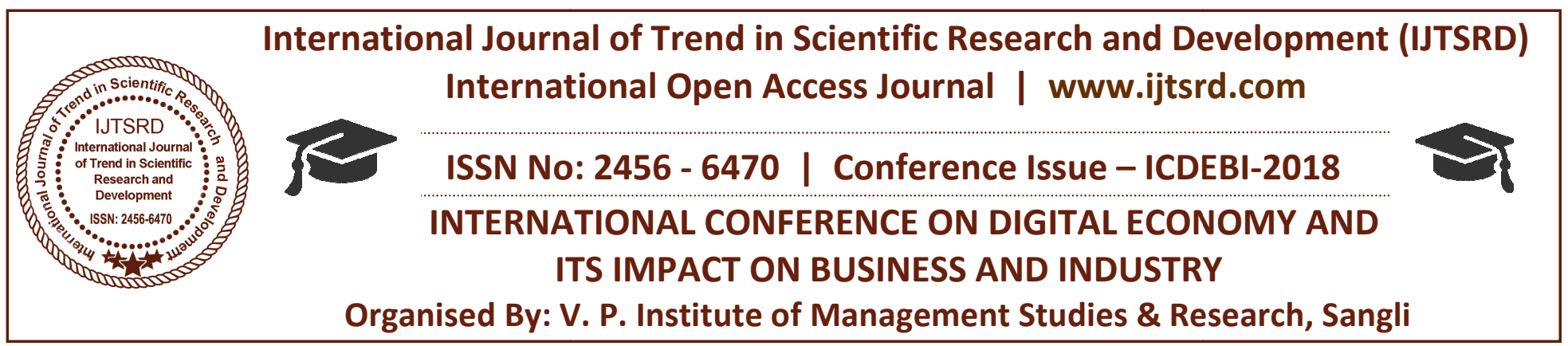

\title{
Digital Storage for Research: Issues and Challenges
}

\author{
Prof. Sudhir Vaijanathrao Panchagalle, Dr. Ravindra Dadarao Gaikwad \\ Assistant Professor, Department of Commerce Shri Madhavrao Patil Mahavidyalaya, \\ Murum, Dist Osmanabad, Maharashtra, India
}

\begin{abstract}
Cloud computing i.e. digital storage is set of resources and services presented through the Internet. Cloud services are delivered from data centers situated all over the world. Cloud computing facilitates its clients by providing essential resources via internet. General model of cloud services is Google apps, provided by Google and Microsoft SharePoint. The speedy growth in field of "cloud computing" also increases strict security concerns. Security has remained a stable issue for Open Systems and internet, when we are talking about safety cloud really suffers. Lack of safety is the only obstacle in broad acceptance of cloud computing. Cloud computing is bounded by many security issues like securing data, and investigative the utilization of cloud by the cloud computing vendor. This paper introduces a comprehensive examination of the cloud computing safety issues and challenges focusing on the cloud computing types and the service release types. This paper mainly proposes the core idea of secured cloud computing. It suggests the cloud computing based on disconnect encryption and decryption services from the storage space service. Due to this increasing require for more clouds there is a still growing risk of security becoming a main issue. This paper shall look at ways in which security intimidation can be a hazard to cloud computing and how they can be avoided.
\end{abstract}

KEYWORD: Computer, cloud computing, security etc.

\section{INTRODUCTION}

The cloud computing becomes the crowd issue in business and academia with the speedy expansion of computer hardware and software. The cloud computing is the outcome of many factors such as conventional computer technology and announcement technology and business form. It is based on the network and has the arrangement of service for the customer. The cloud computing system provides the service for the consumer and has the nature of high scalability and dependability. Cloud computing\| just means, Internet computing, generally the internet is seen as compilation of clouds; thus the word cloud computing can be definite as utilizing the internet to offer technology enabled services to the people and organizations. Cloud computing enables patrons to access resources online through the internet, from wherever at any time without disturbing about technical/physical running and safeguarding issues of the original resources. Besides, Resources of cloud computing are lively and scalable. Cloud computing is independent computing it is completely dissimilar from network and usefulness computing. Google Apps is the supreme example of Cloud computing, it enables to access services via the browser and deployed on millions of machines. Nowadays, we have three types of cloud environments: community, personal, and Hybrid clouds. A community cloud is normal model which providers make several resources, such as applications and storage space, available to the public. Community cloud services may be free or not. In community clouds which they are running applications outwardly by large service providers and offers various profit over private clouds. Private Cloud refers to internal services of a industry that is not available for normal people. Essentially Private clouds are a promotion term for an architecture that provides hosted services to exacting group of people behind a firewall. Hybrid cloud is an surroundings that a company provides and controls some resources inside and has some others for public 
utilize. Also there is mixture of private and public clouds that called Hybrid cloud. In this type, cloud supplier has a service that has confidential cloud part which only accessible by expert staff and protected by firewalls from outside accessing and public cloud surroundings which outside users can access to it.

\section{TYPES OF CLOUD COMPUTING}

There are three main types of service in the cloud environment: SaaS, PaaS, and laaS [1]. In cloud, similar to every future technology, there are some issues which concerned it and one of them is RAS issue. For having good and high presentation, cloud provider must meet several management features to ensure improving RAS parameters of its service such as:

\section{$>$ Accessibility management \\ $>$ Access manage management \\ $>$ Susceptibility and trouble management \\ $>$ Patch and pattern management \\ $>$ Countermeasure \\ $>$ Cloud system using and admission monitoring}

Cloud computing, so as to transport a controllable cloud computing services to the governments, enterprises and persons without the safety danger. unluckily, there are only imperfect labors towards focusing on cloud computing safety on behalf of operator. It is so necessary to behavior a series of technical researches on cloud security from the viewpoint of operators, while pouring the development and introduce it to the business. This paper presents safety problems encounter in cloud computing, and has a investigate on many technical solutions for cloud security evils.

\section{CLOUD SECURITY ISSUE}

Cloud computing and web services run on a system structure so they are open to system type attacks. One of these attack is the dispersed refutation of service attacks. If a user could take control a server then the hacker might stop the web services from performance and order a ransom to put the services back online. To stop these attacks the use of cookies and limiting users linked to a server all help stop a DDOS attack. Another such assault is the man in the center attack. If the secure sockets coating (SSL) is wrongly configured then customer and server verification may not act as expected so leading to man in the center attack. It is obvious that the security issue has play the most significant role in hindering Cloud computing. Without hesitation, putting your data, organization your software at someone else's solid disk using someone else's CPU appears intimidating to many. Well-known safety issues such as data loss, phishing, and botnet pose serious threats to organization data and software. Moreover, the multi-tenancy reproduction and the pooled computing resources in cloud computing has introduced new security challenged that require novel technique to tackle with.

\section{CLOUD SERVICE PROVIDER ISSUE}

Service Provider Security Issues The public cloud computing surroundings offered by the cloud supplier and make sure that a cloud computing resolution satisfies organizational security and privacy needs. The cloud supplier to provision the safety controls necessary to safeguard the organization's information and applications, and additionally the proof provided regarding the effectiveness of these controls migrating organizational information and function into the cloud.

\section{PRIVACY:}

Privacy is the one of the safety issue in cloud computing. individual information system vary across the world and number of limitations located by number of countries whether it stored outside of the nation. For a cloud service supplier, in every jurisdiction a solitary level of service that is satisfactory. Based on contractual commitment data can store within exact countries for privacy regulations, but this is hard to confirm. In case of Private and secret customer's data rising for the consequences and potential costs of mistake for companies that handle. But professionals develop the security services and the cloud service privacy practices. An effective assessment strategy must cover data protection, acquiescence, privacy, identity management, secure operations, and other related security and legal issues. 2.3Securing Data in broadcast Encryption technique are used for data in transmission. To provide the protection for data only goes where the client wants it to go by using verification and integrity and is not modified in broadcast.

\section{SSLLTLS PROTOCOLS:}

SSLITLS protocols are worn here. In Cloud environment nearly all of the data is not encrypted in the processing time, but to process data, for any function that data must be unencrypted. In a fully homomorphism encryption scheme advance in cryptography, which allows data to be processed 
without being decrypted. To give the privacy and integrity of data-in-transmission to and from cloud provider by using admission controls like authorization, authentication, auditing for using resources, and ensure the accessibility of the Internetfacing possessions at cloud supplier.

The cloud system is successively in the internet and the security troubles in the internet also can be originate in the cloud system. The cloud system is not different the traditional system in the PC and it can meet other special and new security problems. The main concerns about cloud compute are safety and privacy. The traditional security evils such as security vulnerabilities, virus and hack attack can also make intimidation to the cloud system and can lead more serious results since of property of cloud computing. Hackers and malicious burglar may hack into cloud accounts and steal sensitive data store in cloud systems. The data and business application are stored in the cloud center and the cloud scheme must protect the resource carefully. Cloud computing is a knowledge evolution of the widespread adoption of virtualization, service oriented architecture and utility computing. over the Internet and it includes the applications, platform and services. If the system meets the breakdown, fast recovery of the resource also is a problem. The cloud systems hide the details of service completion knowledge and the organization. The user can't control the progress of deal with the data and the user can't make sure the data refuge by themselves. The data resource storage space and operation and network transform also deals with the cloud system. The key data resource and privacy data are very introduce for the user. The cloud must provide data control system for the user. The data security review also can be deploying in the cloud system. Data difficult to any authorized place you need it, in a form that any authorized application can use it, by any authorized user, on any approved device. Data uprightness requires that only approved users can change the data and Confidentiality means that only official users can read data. Cloud computing should provide strong user access control to strengthen the licensing, certification, quarantine and other aspects of data management. In the cloud computing, the cloud provider system has many user in a dynamic reply to altering service needs. The users do not know what position the data and do not know which servers are processing the data. The user do not know what network are transmit the data because the flexibility and scalability of cloud arrangement. The user can't make sure data solitude operated by the cloud in a private way. The cloud system can deploy the cloud center in different area and the data can be stored in different cloud node. The different area has different law so the security running can meet the law risk. Cloud computing service ought to be better in legal guard

\section{CLOUD ARCHITECTURE}

All Cloud computing is a set of IT services that are provide to a customer over a arrangement on a leased basis and with the aptitude to scale up or down their overhaul supplies. Usually cloud computing services are delivering by a third party supplier who owns the infrastructure. It advantages to mention but a few include scalability, pliability, litheness, efficiency and out sourcing non-core activities. Cloud computing offer and pioneering commerce model for organizations to adopt IT services without frank investment. There are two basic cloud models are discussed, first the Cloud service model and the subsequent Cloud Deployment model. A. Cloud Service Model Cloud computing is a freedom of computing where extremely scalable IT-related capability are provided - as a service crosswise the internet to numerous external clients. This term efficiently reflects the different facets of the Cloud Computing example which can be establish at different communications levels. Cloud Computing is broadly secret into three services: - IaaS", "PaaS" and "SaaS". Cloud Computing have some different helpfulness services.

\section{CLOUD SERVICE MODEL:}

Cloud Service Model Cloud computing is a release of computing where especially scalable IT-related capability are provided - as a service transversely the internet to numerous external clients. This term effectively reflects the different facets of the Cloud Computing example which can be found at different infrastructure levels. Cloud compute is broadly classified into three services: - IaaS", "PaaS" and "SaaS". Cloud Computing have some dissimilar usefulness services.

\section{IAAS (INFRASTRUCTURE AS A SERVICE) MODEL:}

The main idea behind this model is virtualization where user have practical desktop and consume the resources like network, storage space, virtualized servers, routers and so on, complete by cloud service provider. Usage cost are intended per CPU hour, data 
GB store per hour, network bandwidth inspired, network communications used per hour, value additional services used, e.g., monitor, auto-scaling etc. Examples: Storage services provided by AmazonS3, Amazon EBS. Computation services: AmazonEC2, Layered tech and so on.

\section{PAAS (PLATFORM AS A SERVICE) MODEL:}

It refers to the environment that provides the runtime environment, software consumption framework and constituent on pay to enable the direct deployment of application level property or web application. PaaS is a platform where software can be developed, tested and deployed. It means the entire life cycle of software can be operating on a PaaS. This service model is dedicated to application developers, testers, deplorers and administrators. Examples: Google App Engine (GAE), Microsoft Azure, IBM Smart Cloud, Amazon EC2, salesforce.com and jelastic.com.

\section{SAAS (SOFTWARE AS A SERVICE):}

Through this service release model end users consume the software request services directly over system according to on-demand basis. For example, Gmail is a SaaS where Google is the provider and we are consumers. Other well known examples of PaaS include billing services provided by Arial system, op basis. Financial services: Concur, workday, Backup and recovery services and so on

\section{CONCLUSION}

In this study dissimilar security and privacy related explore papers were calculated briefly. Cloud services are used by both better and smaller scale organization. Compensation of Cloud computing are huge. But it's a global happening that all in this world has advantages as well as disadvantages. Cloud computing is pain from severe protection threats from user point of view, one can say that lack of security is the only worth mention disadvantage of cloud computing. Both the Service providers and the clients must work together to guarantee safety and security of cloud and data on clouds. Mutual indulgent between service providers and users is enormously essential for as long as better cloud security. In this paper we have identified that security is main hurdle in wide receipt of cloud compute. Users of cloud services are in fear of data loss and privacy. Researchers and IT security professional must come onward and do more to ensure security and privacy to users. Our study identify top security concern of cloud computing, these concern are Data loss, escape of Data, Client's trust, User's Authentication, Malicious users handling, Wrong usage of Cloud computing and its services .Hijacking of sessions while accessing data. We propose to use The Cloud Security Alliance (CSA) discharge of a new governance, risk management, and compliance stack for cloud computing. The suite of cloud security tools, available for free download, is meant to help organization create municipal and private clouds that comply with manufacturing standards for conventional governance, risk, and compliance (GRC) best practices. The GRC stack has three components: a technical foundation, a controls framework, and a questionnaire for assess what the CSA calls "industry-accepted ways to file what safety.

\section{REFERENCES:}

1. Bowman, S. Roschke, et aI., "Intrusion Detection in the Cloud," presented at the Eighth IEEE International Conference on Dependable, AutonomIc and Secure Computing, Chengdu, China, 2009.

2. Brodkin. (2008). Gartner Seven cloud computing security Available: http://www.networkworld.com/news/200S!07020 Scloud.html.

3. Ponemon, "Security of Cloud Computing Users," 2010.

4. T. Mather. (2011). Data Leakage Prevention and Cloud Computing. Available: 